\title{
ACIERTOS Y DESACIERTOS DEL SERVICIO DE POLICÍA EN EL ÁMBITO RURAL DEL DEPARTAMENTO DE SANTANDER, COLOMBIA*
}

\author{
RIGHTS AND WRONGS OF THE POLICE SERVICE IN THE RURAL AREA OF \\ THE DEPARTMENT OF SANTANDER, COLOMBIA
}

\author{
Fernando Albeiro Gutiérrez-Otálvaro** \\ Carmen Sofía Cañas-Cañas*** \\ EdWin Estiven Sacristán-Carrillo****
}

\section{Resumen}

Objetivo. Evaluar aciertos y desaciertos del servicio de seguridad rural en el departamento de Santander, Colombia. Metodología. Paradigma cualitativo, privilegiando un enfoque participativo con diseño de investigación acción a través de tres grupos focales distribuidos en: a) sociedad civil involucrada, b) actores de las entidades territoriales relacionados con la seguridad y c) oficiales activos de carabineros con responsabilidad de seguridad rural de cada provincia de Santander. Resultados. Se identificó que esta especialidad del servicio de policía en su proceso de profesionalización debe acentuar la formación de policiales con vocación hacia la ruralidad, el trabajo comunitario, productivo y énfasis en habilidades para la resolución de conflictos. Conclusión. El número de uniformados dedicados a esta actividad en este sector del país es insuficiente y usualmente son ocupados en labores que no estás asociadas a su perfil.

Palabras clave: carabineros, seguridad rural, aciertos, desaciertos, policía.

\section{Abstract}

Objective. To evaluate the rights and wrongs of the rural security service in the Department of Santander, Colombia. Methodology. Qualitative paradigm, privileging a participatory approach with action research design through three focus groups distributed as follows: a) civil society involved; b) actors of territorial entities related to security; and c) active police officers involved with mounted police, with responsibility for rural security in each province of Santander. Results. It was identified that this specialization of the police service should enhance, in its professionalization process, the training of police officers with a vocation towards rurality, productive community work and emphasis on conflict resolution skills. Conclusion. The number of uniformed police officers dedicated to this activity in this region of the country is insufficient and they are usually employed in tasks that are not associated with their profile.

Key words: mounted police, rural security, rights, wrongs, police.

\footnotetext{
* El presente artículo es una reflexión derivada de la investigación titulada "Evaluación de Aciertos y Desaciertos en el Servicio de Seguridad Rural en el Departamento de Santander 2017”, desarrollada en la Escuela de Carabineros de Vélez, Mayor General; Manuel José López Gómez, que pretendió una mirada evaluadora sobre el servicio de seguridad rural prestado por la Policía Nacional colombiana en el departamento de Santander, Colombia, haciendo un recorrido bibliográfico y análisis del modelo de seguridad actual contrastado y enriquecido con las diversas opiniones de los actores directos.

${ }^{* *}$ Escuela de Carabineros de Vélez, Mayor General Manuel José López Gómez. Vélez, Colombia. E-mail: esvel.gruin-jefat@policia. gov.co (D) orcid.org/0000-0002-2670-4167 Google Scholar

**** Escuela de Carabineros de Vélez, Mayor General Manuel José López Gómez.Vélez, Colombia. E-mail: esvel.gruin-jefat@policia. gov.co (1) orcid.org/0000-0001-7893-4688 Google Scholar

***** Fundación Universitaria de San Gil, UNISANGIL. Chiquinquirá, Colombia. E-mail: esacristan22@gmail.com

(D) orcid.org/0000-0002-9316-1182 Google Scholar
} 


\section{Introducción}

El servicio de seguridad en el ámbito rural implementado por la Policía Nacional de Colombia tiene retos y compromisos con las comunidades a propósito de su vulnerabilidad en las situaciones generadas por los actores disidentes de los desarmes en la actual, si se quiere, confusa pos-guerra. Teniendo en cuenta que la policía rural especializada no es suficiente para cubrir toda la geografía nacional distante, y que aunque la comunidad confía en la institución policial, no siempre acude a solicitar sus servicios por la falta de pie de fuerza en sus territorios para atender las necesidades de seguridad ciudadana. Resulta de gran importancia entonces, estudiar las situaciones que han dificultado el posicionamiento, y control de la seguridad rural de la nación y ha propiciado la emergencia de nuevos escollos de violencia ejercida por grupos al margen de la Ley.

En el contexto, de las mayores necesidades de las zonas rurales de alto riesgo están la administración de justicia, la seguridad de la población y el tratamiento de las economías ilegales. Por lo mismo, de las demandas surgidas en el posconflicto son el mejoramiento de los mecanismos para el ofrecimiento de garantías del estado en el ámbito rural. En este sentido, en algunos municipios hacen presencia organizaciones estatales o respaldadas por el gobierno como casas de justicia, comités de convivencia y centros de conciliación, pero al igual estas, otras ilegítimas también han estado presentes ejerciendo roles de procuración de "justicia" ejercidas por grupos armados.

De acuerdo con lo expuesto, con un diseño científico social participativo de actores policiales, civiles y estatales, se hizo necesario responder a la pregunta de investigación: ¿Cuáles son los aciertos y desaciertos del Servicio de Policía Rural en el departamento de Santander, Colombia?, en busca de estrategias de mejora acordadas y horizontales de campo en la aplicación del SISER, aspectos que enriquecieron el desarrollo de políticas de seguridad no solo bajo la percepción del Estado (Ministerio de Defensa), sino con las percepciones de las comunidades en sus territorios a través de la consolidación de aciertos y la disminución de los efectos en los desaciertos identificados.

\section{El servicio de policía en el ámbito rural en Latinoamérica, hacia los retos en Colombia (antecedentes teóricos)}

Al tomar como referencia algunos antecedentes latinoamericanos, principalmente en Brasil, Chile, México y desde luego en Colombia; se establece que en estas naciones el tema de la seguridad, y particularmente en las zonas no urbanas, viene siendo abordado desde otrora, representando un profundo interés en esta área, incluso a pesar de la migración de las poblaciones rurales hacia las urbes. Sin embargo, Colombia presenta datos que hacen esta discusión central 
Aciertos y desaciertos del servicio de policía en el ámbito rural del departamento de Santander, Colombia

y prioritaria para garantizar el orden, goce y disfrute de los derechos humanos en los lugares no considerados céntricos del país: "De acuerdo con el Departamento Nacional de Planeación (DNP, 2015) la vida rural en Colombia sigue teniendo un peso elevado: primero, el 60\% de los municipios colombianos son considerados rurales" (DNP como se citó en Villegas et al., 2016, p. 4).

Teniendo en cuenta, desde luego, el contexto de cada nación, la época, sus nociones de Estado y su filosofía institucional en términos policiales, se identifica que, por ejemplo, en México el interés por la seguridad de la ruralidad se dio "en el año 1826, con el establecimiento de la policía rural por el gobernador Lucas Fernández. Con el establecimiento de la policía rural se conformaron secciones municipales rurales según las diferentes necesidades que se presentaran en el campo" (Rodríguez, 2015, p. 222). Se entiende desde allí que era necesario para asegurar los ideales del Estado comprender las necesidades en términos de seguridad en el campo, al asumir como fundamental la presencia del Estado, no solo a través de lo que podría asemejarse a un corregidor para Colombia en la época, también para que dicho líder estuviera respaldado por una fuerza armada en los rincones de esa patria.

Al igual que en aquella época, hoy la práctica de muchos profesionales de policía está afectada, entre otras cosas del contexto. Asunto que se explica históricamente en parte desde la experiencia mexicana de la seguridad rural en la expresión "Pan o palo" Tal vez no con el imaginario del deber ser hoy, en el actuar de las instituciones policiales en América Latina. No obstante; estos antecedentes ayudan a establecer más allá de lo que se consideraba la garantía de la presencia de la fuerza pública en los sitios distantes, la idea de que, para conseguir y concebir ideológicamente el control bajo una sola idea de Estado es necesario llegar a todos los rincones del territorio.

Por eso, más allá de los imaginarios del General Mexicano Porfirio Díaz, lo que sobrevivió fue la emergente labor comunitaria y de seguridad de las fuerzas policiales en todos los rincones de esa nación bajo una sola lógica estatal. Finalizado el periodo conocido como el "Porfiriato" en el año 1910, los rurales no desaparecieron dado que mantenían la preocupación por la seguridad y cambiaron su denominación a "defensas rurales", con ello dieron continuidad a la labor comunitaria y de seguridad en las zonas rurales, lo que refleja la importancia de esa actividad policial requerida en las zonas vulnerables y de difícil acceso.

Hoy en día México y Colombia comparten complejas situaciones de seguridad rural por cuenta del narcotráfico y el control de sus territorios rurales, debido a la fertilidad de sus tierra, la idoneidad de su clima, su topografía quebrada y de difícil acceso, además de una ubicación

\footnotetext{
${ }^{1}$ Se reconoce a partir del texto titulado ¡Pan o Palo! El perfil social de los jefes de policía rural en Tamaulipas, 1888-1910, dado a conocer en el texto la expresión, Pan o Palo: Pan para el ejército, pan para los burócratas, pan para los extranjeros y hasta pan para la iglesia; y palo para los adversarios y disidentes de don Porfirio (Santoni como se citó en Rodríguez, 2015, p. 223).
} 
geográfica estratégica como puerta de entrada o salida de Sur América hacia Centro América. A su vez, Colombia y México presentan altos índices de violencia en las zonas periféricas a las urbes. "El tráfico de cocaína a Estados Unidos, promovido a través de contactos colombianos y pactos con otros cárteles, como el de Sinaloa" (Maldonado, 2012, p. 24); este es un escenario en el que también, estratégicamente, en aspectos de seguridad rural en las dos naciones se aplican acciones conjuntas entre militares y policiales, entonces en México "una de las estrategias para opacar el movimiento neocardenista fue la profundización de las campañas del ejército y la policía para combatir el narcotráfico regional” (Maldonado, 2012, p. 21).

Por otro lado, en Chile también se destaca la reflexión que coloca al país en los escenarios de circulación científica del conocimiento latinoamericano como sobresaliente por la promoción del control y sobre todo la garantía de la seguridad para sus pobladores.

Desde su creación en 1927, hace 90 años, la policía de Chile ha sido valorada como una de las mejores policías de América Latina; igualmente, "carabineros de Chile es una de las instituciones públicas mejor evaluada por los chilenos” (Oviedo, 2007, p. 73). Esto gracias al nivel de confianza que la comunidad chilena tiene en la misma, siendo este tal vez uno de los mejores escenarios para proyectar los intereses de una institución en el seno coyuntural de su nación. Se da paso a la idea de que los servicios de policía rural no solo deben estar sometidos al juicio y la reflexión del país, sino también a experiencias similares en las naciones vecinas en aras de ampliar la internacionalización de las actividades policiales. Es así como

En el año 1998, Carabineros de Chile implementó una modalidad de servicios policiales preventivos que llamó Plan Cuadrante de Seguridad Preventiva, destinado a brindar seguridad, buena convivencia social y generar mayor confianza, así como a solucionar problemas que aquejaban a la comunidad. (Oviedo, 2007, p. 75)

Así, "los modelos foráneos bien pueden inspirarnos para reestructurar nuestro servicio de policía en el ámbito rural. De hecho, así ocurrió en 1936 con la asistencia técnica de una misión chilena para la organización de la Policía Rural Montada” (Torres, 2015, p. 1). Antecedente que debe alentar estas discusiones no hacia la unificación de criterios ni a la homogeneidad de prácticas, estas aseveraciones no serían pertinentes porque los proyectos de nación son distintos; mejor aún, porque las experiencias exitosas como las no provechosas deban ser tenidas en cuenta para tomar ventaja de sus referentes para la toma de decisiones.

En el caso de Brasil, se estableció un constructo teórico reflexivo a partir de la garantía de la seguridad en un contexto más amplio que se asume desde la empatía que debe emerger en los procesos y políticas de seguridad, pero también desde la premisa institucional que sugiere que esta asuma "que la actividad policial nace de la comunidad, integra a la comunidad y sobrevive 
Aciertos y desaciertos del servicio de policía en el ámbito rural del departamento de Santander, Colombia

en función de la comunidad, destinataria de un servicio público considerado y visto como indispensable para la preservación del orden colectivo" (Souza como se citó en Ribeiro, Neiva e Oliveira y Alves Diniz, 2016, p. 609).

Bajo esta lógica, los brasileños establecieron esquemas de acercamiento a las poblaciones suburbanas y distantes, una modalidad de policía comunitaria llamada "Grupo Especial de Patrullaje” en áreas de riesgo no solo para pretender la presencia de la fuerza bélica del Estado, también para acercar esa fuerza más que para normalizarlos, para respaldarlos y garantizar sus derechos en cada una de sus vivencias.

Todo esto en una estructura sistémica de pilares para potenciar esta cohesión entre comunidad y fuerza policial, así "Brasil crea cuatro iniciativas llamadas Bases Comunitarias, Bases Comunitarias Móviles, Policía y Familia y Red de Vecinos Protegidos, las que tienen como objetivo crear relaciones entre la policía y la Comunidad” (Ribeiro et al., 2016, p. 611). Esto da fuerza al interés por el Estado que no solo como se anticipaba en las anteriores líneas se afanaba por la seguridad del territorio, en este referente se identifica entonces que también se preocupa porque la comunidad respalde esa fuerza, se exprese con agrado de ella e incluso haga parte de la misma sin uniformarse.

Sin embargo, se anticipa que no necesariamente la presencia de la fuerza pública garantiza la sinergia buscada, además porque la disciplina castrense, el rigor del cumplimiento y otros que podrían llamarse prácticas usuales de las instituciones de este corte no son de fácil adaptación a las dinámicas de las comunidades. Se indica entonces que las acciones de los policías de Belo Horizonte son represivas, que no buscan el acercamiento con la comunidad y, en últimas instancias, las estrategias aplicadas por estos son solo las mismas actividades que conllevan al uso de la fuerza pero bajo la legalidad que les atribuye el vocablo policía comunitaria (Ribeiro et al., 2016, p. 633).

Luego de referenciarnos en perspectivas de gran interés en los antecedentes de algunas naciones representativas en Latinoamérica, nos permitimos establecer que algunas de las prácticas que se desarrollan en las políticas de seguridad rural en Colombia han tenido diálogo con las reflexiones de otras naciones para emprender una identidad sobre conceptos tan significativos como la garantía de la seguridad y los derechos humanos en las zonas rurales.

La ruralidad en Colombia goza de diferentes escenarios de complejidad circundados por las distinciones de su población, etnias, geografía, hidrografía, clima y costumbres, entre muchos elementos que van marcando un panorama de difícil interpretación y abordaje imposible a través de una postura homogénea de las necesidades del territorio nacional en cuanto a seguridad; sin embargo, a través de la experiencia de la Policía Nacional y seguridad ciudadana en el Estado, intenta ofrecer un concepto para la comprensión de dicha particularidad: "La 
ruralidad es la representación de las relaciones sociales gestadas en un espacio geográfico, que se caracteriza por la dispersión, baja densidad de la población y la distancia con respecto de las cabeceras y los bienes y servicios que ofertan” (Villegas et al., 2016, p. 4).

Esto transversalizado por la pacificación del territorio en el término de un conflicto interno que superó las cinco décadas y que ofrece retos en este nuevo proyecto de nación en medio de un ambiente enrarecido por las disidencia ${ }^{2}$, es lo que constituye esta nueva realidad. La Policía Nacional colombiana ha establecido, erigido y presentado al país el proyecto para el posconflicto el "Plan Estratégico Institucional, Comunidades Seguras y en Paz 2015-2018 y la visión 2030" y a su vez anclado en su centro el "Sistema Integrado de Seguridad" (SISER) como "un modelo de coordinación interinstitucional e intra-institucional que busca mantener un engranaje al servicio del sector rural colombiano" (Villegas et al., 2016, p. 1). Como una respuesta consecuente con las necesidades que se vislumbran al momento de sostener y retomar el control del territorio ocupado por fuerzas armadas al margen de la ley.

Sin embargo, este plan y sistema se encuentra inmerso en lo que podría denominarse la complejidad rural en Colombia, asunto que no es menor en cuanto a que sus actividades no se someten a la reflexión académica, y más aún a la discusión científica, puede llevar el intento de proveer seguridad en el territorio nacional a una experiencia no exitosa y recaer en la prolongación de conflictos sectoriales o, peor aún, organizados cuando es unidireccional y no se acuerda con los territorios.

El SISER ${ }^{3}$ presenta seis principios generales que pretenden atender la complejidad y la necesidad ya esbozada, bases en las que se enumeran sus elementos más importantes: 1) respeto por los derechos humanos y la diversidad, 2) corresponsabilidad, 3) cobertura, 4) protección, 5) priorización y progresividad y 6) participación ciudadana (Villegas et al., 2016, p. 13). Estos pilares son orientados, liderados y administrados, principalmente, a través de los fuertes de carabineros que coordinan y desarrollan las actividades.

A partir de estas consideraciones, se promueven estrategias para la consolidación de la seguridad rural, la presencia del Estado y las garantías en términos de derechos humanos que deben asegurarse para toda la comunidad ubicada en la periferia del país, las Unidades Básicas de Carabineros - UBICAR - constituyen una gran proyección de la seguridad rural a corto, mediano y largo plazo, al ser estas las encargadas de atender los cuadrantes con alcance rural y los

\footnotetext{
${ }^{2}$ Con la firma de los primeros acuerdos de cese bilateral al fuego entre el gobierno colombiano y las FARC, se convierten en una excepcional oportunidad para repensar y reemprender la construcción de la nación colombiana sobre bases territoriales más genuinas, auténticas y pertinentes (Montañez, 2016, p. 11).

${ }^{3}$ En el nivel táctico, territorial, el SISER opera a través de los comandos de región y departamento, en donde se encuentran las regionales de carabineros, desde las cuales se planifican las acciones policiales de intervención rural por intermedio de los fuertes de carabineros, en complementariedad con las regionales o coordinaciones de inteligencia, investigación criminal, de antisecuestro y antiextorsión y los centros estratégicos operacionales de antinarcóticos, CEO (Villegas et al., 2016, p. 20).
} 
Aciertos y desaciertos del servicio de policía en el ámbito rural del departamento de Santander, Colombia

segmentos estratégicos” (Villegas et al., 2016, p. 21); así estrategias como el plan cuadrantes se ha implementado incluso bajo la perspectiva de experiencias foráneas como las chilenas.

Gracias al respaldo gubernamental, la Policía creó una estrategia de patrullaje denominada "Plan Cuadrante" que ha tenido algunos factores favorables, pero a su vez ha ocasionado que el enfoque de la función de la policía cree debate entre la prevención y el control, ya que se requiere "contundencia" con la delincuencia en general, pero al mismo tiempo que logre evitar que se generen los escenarios propicios para que desemboquen en hechos que afecten la seguridad.

Estrategias que han dejado pautas para la construcción de diseños que atiendan las necesidades del territorio nacional; no obstante, se anticipa una amplia reflexión del deber ser en términos de seguridad rural a partir de un modelo que goza de elementos eclécticos pero que debe ser sometido a la rigurosidad permanente de la deconstrucción y reconstrucción de la mano de la ciencia, la tecnología y la participación comunitaria. Se asume la seguridad rural como una apuesta que va más allá de la mera presencia de una fuerza armada, atribuyéndose policialmente el protagonismo en el proyecto de Estado que corresponde a que "la Policía sería el centro de la idea moderna de Estado, al garantizar la libertad y la seguridad individuales por medio de la vigilancia constante contra cualquier uso indebido de la fuerza, ya sea interno o externo" (Weber, 1994, p. 25).

Lo que constituye un verdadero reto para las instituciones policiales asumir este liderazgo en el que seguramente también resulta importante el uso de la fuerza que además es intrínseco al servicio de la policía; sin embargo, emergen otras competencias de las que son objeto también los estudiantes en las escuelas de carabineros, entre ellos el liderazgo comunitario, emprendimiento, empoderamiento y desarrollo de la sensibilidad para asegurar que la comunidad confíe en la presencia del Estado a través de los miembros policiales, siendo la confianza uno de los pilares fundamentales de cualquier sociedad.

Entonces hablamos de una institución armada que asume roles que hasta ahora muchas comunidades podrían considerar desconocidos o no usuales, ya que a los policías por otras épocas se les reconocía como una organización que tenía como su primer instrumento de intervención la fuerza, los nuevos retos requieren que la fortaleza en su actuar sean principalmente sus habilidades de mediación, que faciliten a través de la educación y la convivencia ciudadana, dejando estrictamente el uso de la fuerza como última opción ${ }^{4}$. En este sentido, entre más se documenten ejercicios sociales del liderazgo de policiales carabineros

\footnotetext{
${ }^{4}$ Hay que comprender que la institución de policía está orientada a la preservación de la convivencia, lo que implica comprender la cultura, el comportamiento de la sociedad y la protección de los recursos; es una institución mediadora, para lo cual se apoya en la educación y la prevención, teniendo como último recurso la fuerza; su fundamento es la ciencia de policía, que es una disciplina autónoma de la civilidad jurídica de la convivencia ideal humana (Londoño, 2011, p. 25).
} 
con las comunidades rurales, emergerán elementos propios territoriales para las mejoras de la seguridad en cada caso.

Asumiendo entonces como principal premisa dentro de las lógicas de la seguridad rural que "las respuestas a las nuevas demandas de seguridad no solo pueden provenir de un cuerpo de policía, sino de la construcción de saberes sociales que propicien la convivencia” (Habermas, 2002, p. 32). Lo que supone es imperativo el empoderamiento de las comunidades de su misma tranquilidad y, por tanto, de su seguridad, tomando como referente el conocimiento de normas básicas para el cumplimiento en el que están involucrados todos los actores, perspectiva en que los policiales resultan ser solo un miembro de esa convergencia, en tanto que son las poblaciones quienes lideran el camino hacia la seguridad rural.

\section{Diseño metodológico y paradigma científico}

De acuerdo al objeto de estudio, la investigación desarrollada se enmarcó en el paradigma cualitativo en el que se proyectó una apuesta metodológica que interpretara el fenómeno social estudiado y que abordara los aciertos y desaciertos del servicio de policía en el ámbito rural del departamento de Santander, Colombia. El proceso metodológico pretendió comprender y afectar constructivamente el fenómeno estudiado proponiendo mejoras en la puesta en marcha del servicio de policía en aras de alcanzar una inmersión de las opiniones e ideas de todos los actores, en el proceso que generará confiabilidad en temas de seguridad para las poblaciones, así como involucrar escenarios en que los miembros civiles del proceso de seguridad se empoderarán de los alcances de las estrategias puestas en marcha por la Policía Nacional.

\section{Investigación acción en el interés social de la Policía Nacional}

Debido a que es una iniciativa metodológica con idoneidad para solución de problemas y mejoras en la puesta en marcha de políticas, programas, estrategias entre otros escenarios de intervención. "La investigación Acción como el estudio de una situación social con miras a mejorar la calidad de la acción dentro de ella” (Elliot como se citó en Hernández et al., 2010, p. 509). Todo esto bajo la idea de trabajo mancomunado entre tres tipos de actores inmersos y responsables en la seguridad rural en la búsqueda de construir, con herramientas existentes, estrategias que mejoren la aplicación de los resultados.

\section{Técnicas de recolección de datos cualitativos}

Se realizaron grupos focales bajo el paradigma científico social con el objeto de enlazar miembros políticos-sociales, población civil y agentes de policía de especialidad rural con la intención de fomentar una reflexión en torno a los resultados positivos y negativos que han 
Aciertos y desaciertos del servicio de policía en el ámbito rural del departamento de Santander, Colombia

generado los principales criterios de aplicación de las estrategias del servicio de policía en el ámbito rural, desarrollados por la Policía Nacional desde la implementación del Sistema Integrado de Seguridad Rural SISER en Santander, Colombia.

\section{Grupos de enfoque y número de sesiones}

Respecto a los grupos de enfoque, denominados así por la tradición sociológica nor teamericana, según lo señala Galindo (1998) en su publicación "Técnicas de investigación en sociedad, cultura y comunicación” o por los grupos de discusión, forma europea, en este ejercicio no pretendieron ser una técnica estricta e inamovible en su proceder, aunque rigurosamente si guardan diferencias en su concepción e intervención; Galindo (1998) sugiere entonces que "en la forma Europea el moderador interviene muy poco, usualmente el discurso que produce el grupo no tiene mucha intervención; en la forma norteamericana el moderador controla mucho la participación de cada participante" (Galindo, 1998, p. 76).

El grupo de enfoque fungió como un escenario de recreación de los contextos sociales y pretendió el diálogo e interpretación de las experiencias y análisis de los saberes construidos en diferentes contextos sobre seguridad rural. Dando provecho a que en esta técnica "se trata de ver lo que dicen los sujetos en una situación y en un grupo particular, lo que alguien dice, la manera como lo dice" (Mejía, 2008, p. 61). Para efectos de este proceso, se convocaron tres grupos focales de rangos de asistencia entre seis y nueve participantes por grupo focal; 1) de policías carabineros de especialidad rural asignados a Santander, 2) población rural ubicada en distintas zonas del departamento, quienes poseen experiencia vivida sobre la seguridad en sus territorios y una percepción general sobre el servicio de policía en sus sectores distantes y 3) actores político-administrativos de los entes territoriales relacionados con la seguridad en sus localidades que ofrecieron apreciaciones de las vivencias de seguridad rural en su ubicación a lo largo del departamento.

Los grupos focales se realizaron teniendo en cuenta una caracterización y justificación detallada de sus participantes, con asistencia equilibrada en género y de edades mayores a veinticinco años como criterio que garantiza las vivencias en los territorios, desarrollando una única sesión para cada grupo y de manera diferenciada; cada grupo focal no tuvo contacto con los otros dos tipos de población, de hecho las sesiones se realizaron en fechas distintas. Después de abstraer la información acaecida en las experiencias de la seguridad rural, se prosiguió a interpretar los aciertos y desaciertos que mueven los criterios de dichas prácticas basados en categorías prediseñadas en la apuesta de la seguridad rural, de igual manera abiertos a categorías emergentes, interpretando la información través de la triangulación de datos cualitativos. 


\section{Población objeto de estudio}

La población que participó en los grupos focales desde una perspectiva geográfica del estudio estuvo enmarcada en Santander, departamento que cuenta con 87 municipios en seis provincias, representadas a través de la proveniencia de los asistentes de cada una de las zonas de la ubicación geográfica en estudio para la aplicación de instrumentos en los grupos de enfoque. Las provincias del departamento son las siguientes: Comunera, García-Rovira, Guanentá, Mares, Soto y Vélez. De tal suerte se logró la asistencia desde cada región, siendo los grupos focales la única forma de recolección de información.

\section{Triangulación de datos cualitativos}

En aras de interpretar información recogida y expresada por los participantes en cada uno de los grupos focales, se realizó un ejercicio de contraste triangular de respuestas a partir de preguntas formuladas sobre categorías preestablecidas identificando aciertos y desaciertos del servicio de policía en el ámbito rural teniendo como criterio de hallazgo, las tendencias identificadas en la frecuencia lingüística, descubriendo las convergencias y divergencias desde la mirada de los actores establecidos como población. Ejemplo: Categoría prediseñada, "presencia frecuente de uniformados en una vereda". Sometido a la opinión de los grupos focales conformados por: a). Carabineros; b). Comunidad-civil rural; c). Representantes sector público.

\section{Perspectiva de análisis sobre los resultados de investigación}

Luego de la recopilación de información cualitativa a través de herramientas de recolección como grabadoras de audio y video en las sesiones realizadas, el primer tratamiento realizado a los datos fue la transcripción literal de las narrativas construidas en las discusiones abordadas en las sesiones de los grupos de enfoque. En un segundo momento, se procedió a codificar la información en matrices de análisis de datos cualitativos de acuerdo con categorías prediseñadas en el desarrollo del ejercicio científico; sobre el particular se logró definir las siguientes grandes categorías de análisis: (i) cobertura del servicio de seguridad en el ámbito rural, (ii) corresponsabilidad de las autoridades locales, regionales, nacionales, públicas-privadas y (iii) participación ciudadana.

Aciertos del servicio de policía en el ámbito rural sobre: cobertura del servicio de seguridad en el ámbito rural. Corresponsabilidad de las autoridades locales, regionales, nacionales, públicas - privadas y participación ciudadana.

Tanto población civil rural, policía/ carabineros y autoridades político- administrativas tienen concepciones convergentes positivas sobre la presencia de uniformados, ya sea bajo 
Aciertos y desaciertos del servicio de policía en el ámbito rural del departamento de Santander, Colombia

la organización de las estaciones en los cascos urbanos o directamente de los carabineros, quienes siempre dejaron percepciones acertadas en términos de eficiencia. Esto porque los policiales hacen más segura, tranquila y digna la vida de los sectores apartados de las urbes; esa eficiencia de manera conclusiva deja ver no solo temas asociados a la protección, también emergieron prácticas de estrategias creadas por la experiencia de los profesionales de policía, como facilitar o acercar la atención médica a la comunidad, campañas de prevención del delito, gestiones con instituciones de educación y hasta búsqueda de apoyo en temas productivos. La presencia de uniformados en las zonas rurales es entonces un eje de la labor social alrededor de la seguridad ciudadana.

Uno de los elementos emergentes bajo los aciertos de presencia de los funcionarios de seguridad rural fue el criterio de "agrado", no solo en el reconocimiento por ser ocasionalmente los únicos que llegan a lugares en algunos casos de difícil acceso, particularmente agradan a la comunidad porque el carabinero a diferencia del policía motorizado se hace cercano en una “actitud deferente”, según indican los diálogos, algo que podría nominársele como la estética del carabinero, entre ellos su uniforme, sombrero, cordón y caballo a diferencia de fusil y motocicleta. Los carabineros responsables de la seguridad rural facilitan mejor acceso y acercamiento de las poblaciones al servidor policial, todo esto acompañado de una serie de actividades lúdicas, recreativas y sociales que facilitan la consolidación de un escenario en donde los actores en todas las vías refieren estar satisfechos los unos con los otros.

La confianza que logra el funcionario de seguridad en el ámbito rural resulta ser un elemento intangible producto de la intersubjetividad pero a su vez importante pues se respalda en la idea de hacer evidente que a partir del acercamiento entre unos y otros se construyen proyectos como el trabajo estratégico entre la comunidad con los funcionarios del sistema de seguridad rural; asimismo, son determinantes al momento de consolidar la eficiencia de la seguridad rural, no es posible contar con el apoyo de la comunidad, si la imagen y el acercamiento de los policiales es fría, desconfiada y sin comunicación. Un elemento que contribuye a mejorar y ratificar la percepción de la ciudadanía, ya que siguiendo a Frühling (2009), "la opinión pública demuestra escaza confianza en la policía en el conjunto de la región, con excepción probablemente de las dos policías chilenas, la policía Nacional de Colombia y la policía de Nicaragua" (p. 33).

La corresponsabilidad de actores entendida como la gestión interinstitucional liderada por los funcionarios del servicio de seguridad rural con organizaciones presentes en la periferia como las juntas de acción comunal o cualquier emprendimiento representativo, corresponde a lazos de comunicación que se pueden generar para mantener informado al centro de mando en el área urbana sobre lo que acontece en las zonas distantes, surgiendo de igual manera el empoderamiento de la seguridad como un asunto vital dentro de los aciertos del servicio de policía identificados en los grupos focales, es decir, al gestor o policía a cargo de la seguridad 
rural le corresponde empoderar a estos líderes comunales, emprendimientos organizados, empresas, vigilancia privada y movimientos organizativos para que puedan ser aliados en las estrategias de protección rural comunitaria.

Los entes territoriales son las instituciones que en primer orden se convierten en aliados estratégicos perfectos para cumplir los retos en los servicios de seguridad rural, teniendo en cuenta que regularmente concejales y burgomaestre han sido elegidos popularmente su presencia en las actividades de promoción social de la seguridad juega un papel central ya que antes que a los policiales, la población rural reconoce el liderazgo de las personas que eligieron y les representan. Lo que constituye en acierto las actividades cooperativas por la seguridad que cuenten con la presencia estatal de los gobiernos locales.

Se establece por último, bajo esta categoría de análisis en cuanto a aciertos que las instituciones de naturaleza privada con o sin ánimo de lucro pueden ser fuentes de cooperación para la seguridad rural, ya sea a través de financiamiento de acciones o de cooperación en red para aquellas organizaciones que se encuentran ubicadas en la periferia urbana o de presencia de sus instituciones en las acciones que para la convivencia pacífica programa el servicio de seguridad en el ámbito rural.

\section{Desaciertos del servicio de policía en el ámbito rural sobre cobertura. Corresponsabilidad de las autoridades locales, regionales, nacionales, públicas - privadas y participación ciudadana}

Con gran iteración emerge en los diálogos de los grupos focales la multiplicidad de funciones como un elemento preponderante en los desaciertos del servicio de policía en el ámbito rural, sobresale entonces que en los lugares donde hay presencia de carabineros su dedicación es compartida con actividades múltiples relacionadas con su perfil dentro de la institución; es decir, la de trabajar en el sistema de seguridad rural, así estos funcionarios pasan tiempo en dispersiones desde luego útiles como acompañando a ciclistas, asistiendo a personas en los bancos o en labores administrativas pero reduciendo su dedicación de tiempo central en lo rural. "Los policías comunitarios ya formados son trasladados a otras unidades según las necesidades del servicio. En Colombia, por ejemplo, policías comunitarios son enviados como policías contraguerrilla” (Ruíz, 2004, p. 23).

Aunque la institución policial ha tratado de aumentar la disponibilidad de uniformados para cumplir objetivos de seguridad rural sigue siendo una prelación identificada en las narrativas como un desacierto, esto sustentado en la cifra de treinta y cuatro carabineros ${ }^{5}$ a cargo del sector rural de todo Santander, que aunque cuentan con el apoyo de las estaciones y sus

\footnotetext{
${ }^{5}$ La cifra relacionada fue tomada de las narrativas expresadas en las sesiones de grupo de enfoque como fuente directa.
} 
Aciertos y desaciertos del servicio de policía en el ámbito rural del departamento de Santander, Colombia

comandantes llama la atención en la población objeto de estudio que se agudiza la problemática por la insuficiencia el pie de fuerza. Esto también deja al desnudo y libre de presentar el SISER "un modelo de coordinación interinstitucional e intrainstitucional que busca mantener un engranaje al servicio del sector rural colombiano” (Villegas et al., 2016, p. 1). Como una estrategia interesante pero hasta ahora poco efectiva por falta de personal que respalde sus excelentes planteamientos, según la opinión de los actores de los grupos focales.

También se identificaron como desaciertos del servicio de seguridad en el ámbito rural; dificultades con el apoyo gubernamental, la tenue importancia del carabinero y su servicio dentro de las prioridades en las decisiones de las autoridades administrativas, se concluye en las narrativas la necesidad de que esta especialidad deba ser permanentemente fortalecida, reconocida y articulada reflejándose la contraprestación en otras dimensiones; por ejemplo, en los gastos de movilidad, para lograr mejor cobertura e implementos para el servicio, asuntos que presentan impedimentos para el efectivo desarrollo de las actividades en la periferia de los municipios o lugares apartados.

Muy relacionado con el respaldo gubernamental se encuentra la limitación logística, un criterio elemental que compromete en esencia los ejes del servicio de seguridad en el ámbito rural, los criterios que respaldan este hallazgo dirigen la atención a una organización logística propia de la implementación del servicio de seguridad en el ámbito rural bajo la presencia de carabineros, el esquema no contempla solamente al binomio (carabinero y caballo); es más bien un equipo que acompaña esa actividad para garantizar la eficiencia de las actividades que desarrollan bajo este modelo.

También emerge con gran prelación en los grupos focales la formación académica del carabinero o funcionario del servicio de seguridad en el ámbito rural, elemento que también termina por ser una necesidad en la mayoría de los que trabajan en estas acciones pero que no poseen la formación suficiente. "La aproximación entre policía y comunidad hizo pública la precariedad de los recursos materiales y la ineficacia de la organización policial para resolver los problemas de criminalidad y garantizar la seguridad pública” (Frühling, Beato, Chinchilla, Llorente y De Misquita, 2004, p. 141).

\section{Discusión}

De acuerdo con lo anterior, resulta inerme cualquier consideración de mejora del Servicio de Seguridad Rural si no se tiene una disponibilidad de personal y con los roles dispuestos por el sistema para atender los requerimientos de la seguridad comunitaria de la periferia; siendo necesario el fortalecimiento del número del pie de fuerza de efectivos uniformados con misiones específicas en esas zonas distantes de Santander. Para alcanzar el ideario expresado en la política para la consolidación del servicio de policía en el ámbito rural es preciso interiorizar 
y cumplir con los más altos niveles de exigencia del servicio: "La institución a través de sus diferentes especialidades del servicio en las áreas rurales, desarrollará acorde con su misionalidad, las capacidades necesarias en términos de cobertura, movilidad, comunicaciones y talento humano" (Santos et al., 2010, p. 51).

Atendiendo a la estructura orgánica de la policía nacional, la dirección general imparte órdenes e instrucciones las cuales de manera jerárquica se ejecutan a través de las diferentes direcciones de policía, quienes supervisan, controlan, lideran, deberán asignar funciones y responsabilidades específicas a través de los comandos de distrito, estación y de grupos especializados adscritos a la dirección de carabineros y seguridad rural, haciendo efectivo también acompañamiento positivo a las necesidades en su labor en las veredas o corregimientos.

En efecto, la escasa presencia policial en las veredas se convierte en una situación compleja para su descripción y caracterización, esto sumado a que garantizar permanencia y efectividad institucional según las necesidades y ausencias expuestas en los grupos focales, requiere de interdisciplinariedad diaria con las especialidades de la Seccional de Inteligencia Policial, SIPOL y la Seccional de Investigación Criminal SIJIN entre otras, advirtiendo que en algunas zonas del país existe presencia de grupos al margen de la ley o al menos reductos de estas estructuras criminales, lo cual requiere de especial atención teniendo en cuenta que es un factor que limita la eficiencia en la atención de los casos en términos de tiempo, por cuanto se requiere de una planeación para el desplazamiento, con el fin de reducir riesgos a la integridad física de los uniformados y bienes al servicio del Estado.

Es por esto que las unidades básicas de investigación criminal (UBIC), deben tener actualizada la información de que lo sucede a diario en las veredas (acuerdos cooperados con la comunidad en el área rural), para con ello brindar notificación pormenorizada al cuadrante, para un desplazamiento seguro y efectivo teniendo en cuenta que dichas unidades se encuentran analizando los fenómenos delincuenciales más frecuentes en las ciudades y la forma de desarticular su estructura de una manera mucho más ágil, dejando de lado zonas que por su impacto no requieren una actuación más directa.

En ese mismo sentido, es menester entonces tener en cuenta el factor presupuestal, asunto primordial para el emprendimiento de cualquier proyecto que pretenda impactar la seguridad y convivencia en el sector rural. La financiación de la seguridad ciudadana depende de la capacidad fiscal de los municipios asunto que marca distinciones entre ciudades y municipios consecuentemente, traduciéndose en la cantidad de policías, medios, equipos e instalaciones a disposición de atender la seguridad rural (Bulla y Guarín, 2015, p. 14).

Entonces por la misma organización administrativa de los recursos, aquellos son destinados al departamento de policía o las entidades territoriales y se entiende que deba adoptarse 
Aciertos y desaciertos del servicio de policía en el ámbito rural del departamento de Santander, Colombia

una partida presupuestal específicamente para la seguridad rural que usualmente resulta insuficiente para lograr una cobertura del servicio de seguridad en el ámbito rural eficiente. "Los departamentos y municipios podrán aportar recursos propios o recibir donaciones de particulares destinadas a propiciar y garantizar la seguridad y la convivencia ciudadana” (Ley 1421 de 2010).

Por tal motivo es importante resaltar que es necesario una asignación presupuestal a los comandos de departamento y policía metropolitanas con recursos destinados específicamente al fortalecimiento del sistema de seguridad rural y, de la misma forma, impartir órdenes concretas a los comandantes de departamentos y de estaciones para la elaboración de proyectos encaminados a mejorar la seguridad rural y radicar tales proyectos en las alcaldías y departamentos para el correspondiente aval de los regentes administradores a fin de robustecer los recursos mediante la gestión de recursos de los fondos territoriales de seguridad y convivencia ciudadana, FONSET.

De acuerdo con lo anterior, el panorama para el campesino en materia de seguridad resulta un poco sombrío, pues se requiere de la Dirección de Carabineros y Seguridad Rural, DICAR, volcada completamente al sector rural donde no solo estén dos o tres uniformados cubriendo un caserío, inspección o vereda, pues a pesar de hablarse de posconflicto se presentan distintas formas de violencia y de otros delitos como la minería ilegal, entre otros. Es por eso que se debe reacomodar el servicio de policía en el ámbito rural para dinamizarlo, de tal forma que el ciudadano se sienta no solo acompañado en el mejor de los casos. Sino que sea también actor principal y protagonista en los quehaceres cotidianos como lo hace el modelo nacional de vigilancia comunitaria por cuadrantes.

Lo dicho hasta aquí supone una reflexión sobre la atención más próxima a la resolución de conflictos en el campo como las formas alternativas de superar estas dificultades, pues el impedimento para resolver un caso de forma adecuada siempre va a ser la falta de personal capacitado que cuente con los conocimientos necesarios en materias como infancia y adolescencia, investigación criminal para la indagación y esclarecimiento de delitos contra la vida, la integridad sexual o patrimonio económico, que se presentan con frecuencia en zonas apartadas de una urbe o capital. "Las formas alternativas corresponden a mecanismos no formales y solidarios que brindan un elemento fundamental con la humanización del conflicto, con la presencia de una tercera persona que actúa como facilitadora especialista en resolución o prevención de conflictos" (Fuquen, 2003, p. 274).

Este asunto debe ampliar las competencias de los policías que prestan el servicio de seguridad en el ámbito rural puesto que de seguir sujeto a las especialidades que tiene la policía, el servicio se va a tornar lánguido, parsimonioso, falto de efectividad, pues el hecho de existir cinco o diez policías en una vereda sin los conocimientos adecuados, conlleva a un desgaste de 
personal, apartándose de los criterios de efectividad e inmediatez, ya que siempre se va a estar a la espera de otro funcionario para terminar con una diligencia. "Se trata entonces de educar profesionales de policía formados en valores, técnicas y procedimientos, que además cuenten con la capacidad de proponer y transformar las problemáticas de convivencia y seguridad que afectan a las comunidades" (Ramírez y Céspedes, 2016, p. 198).

Lo relatado no desestima la labor preventiva y comunal del servicio de policía; sin embargo, por las características de los delitos en puntos de alto impacto y sobre los cuales recae el mayor índice delincuencial, conviene entonces disponer de todas las especialidades en una sola a través de un equipo interdisciplinario en una dinámica más frecuente de las UBICAR, contempladas en el SISER para así evitar desgastes tanto procesales como administrativos al momento de recibir una denuncia o de atender un caso sin importar su complejidad, para que se logre dar un tratamiento con la misma eficacia y celeridad como normalmente sucede en las ciudades. "Esto demandará de la Policía Nacional el fortalecimiento de las capacidades institucionales; el esfuerzo del componente educativo y de entrenamiento en las escuelas de formación” (Bello, 2014, p. 331).

La resolución de conflictos en las veredas, caseríos, inspecciones y corregimientos, contienen un ingrediente adicional a la prevención: la inmediatez, puesto que en un sinnúmero de eventos el uniformado no puede llegar a tiempo o dentro de un horario racional después de la ocurrencia de los hechos a conocer la novedad y es ahí donde hay que disminuir esa debilidad, que resulta tan acentuada en estas jurisdicciones. Por ello es imprescindible la presencia permanente del policial no de forma esporádica o por rondas, como se hace en algunos departamentos y que se hace en cumplimiento del SISER, sino como un ejercicio diario, estable, al cual el campesino pueda acudir en cualquier momento, sin dilaciones injustificadas por la ausencia de parque automotor o logístico y atendiendo a principios como el respeto por los derechos humanos; que es transversal a una atención adecuada; sin hacer más gravosa la situación del administrado más que el hecho de que le sea escuchada su denuncia.

Finalmente, no se debe pasar por alto la normatividad existente que regula al servidor público a actuar conforme a lo pretendido por el ciudadano ya que las actuaciones judiciales, ejemplo las diligencias de entrevistas, recolección de información, elementos materiales probatorios, entre otros, que se adelanten en contra del presunto infractor de la ley, frecuentemente se desvanecen por las decisiones judiciales con poca efectividad sobre los indiciados; de igual forma las contravenciones al código de policía que se revisten de bajo poder coercitivo deja desamparado cualquier procedimiento de policía, haciéndose urgente una transformación en tipos penales y normas de convivencia y seguridad ciudadana que se adapten a las circunstancias por las cuales atraviesa el país en torno a pos-conflicto ya que esa mutación de los grupos al margen de la ley desencadena otros tipos de conductas. 
Aciertos y desaciertos del servicio de policía en el ámbito rural del departamento de Santander, Colombia

En la perspectiva de generar una atmósfera de inseguridad y zozobra generalizadas, ello podría ser una estrategia deliberada dirigida a crear confusión, impotencia y desconfianza hacia las instituciones del Estado y los procesos de paz, junto con la negación de la democracia como el escenario propicio para la garantía y efectividad de los derechos fundamentales. (González y Delgado, 2018, p. 6)

Se propone entonces que en el óptimo procedimiento para resolver un conflicto es que el campesino acuda de manera directa al policía y este tenga las herramientas necesarias para atender los diferentes conflictos, haciendo que "la capacitación de las personas que participan en los comités de carácter local sea imprescindible, si se desea que la participación ciudadana supere el mero reclamo por la inactividad policial” (Frühling, 2003, p. 24). Caso contrario deberá acudir a los cuerpos especiales de la policía y hacer un acompañamiento hasta el final del proceso, esto supeditado a leyes del Estado.

\section{Conclusiones}

- Buscar desde la academia a través de la educación continua la capacitación permanente del profesional de policía a cargo de la seguridad rural en procesos formales como la conciliación y la mediación para la solución de conflictos, además de atención a la comunidad, haciendo especial énfasis en la restitución de tierras.

- La resolución de conflictos en las veredas, caseríos, inspecciones y corregimientos, contiene un ingrediente adicional a la prevención y es la inmediatez, para ello se requiere aumento en el número de uniformados atendiendo los conflictos, asignación de recursos para dichas labores y un ejercicio diario, estable, al cual el campesino pueda acudir en cualquier momento, sin dilaciones injustificadas por la ausencia de parque automotor o logístico y atendiendo a principios como el respeto por los derechos humanos.

- Las políticas gubernamentales apuntan al apoyo del agro y de la comunidad rural en busca del regreso al campo de tantas familias desplazadas, víctimas de la violencia, es por ello que desde la institución se han creado programas como Policía de Carabineros, no sólo para la erradicación de cultivos ilícitos, sino para apoyar a la comunidad rural en la puesta en marcha de sus proyectos productivos.

- La participación de la comunidad debe presentarse de forma activa no solo como fuente de información estratégica, el uso de la metodología de investigación-acción presentó durante el desarrollo de la investigación interesantes aportes que nutren la creatividad de lo que puede hacerse en el campo para mejorar la seguridad con la acción de los pobladores a partir de sus saberes. 


\section{Referencias}

Bello, C. (2014). Desafíos y estado futuro de la convivencia en Colombia al 2025. Revista Criminalidad, 56.

Bulla, P. y Guarín, S. (2015). Seguridad Rural en Colombia, una oportunidad para la consolidación del Estado. Recuperado de http://cdn.ideaspaz.org/media/website/ document/55e0c4e3e93c2.pdf

Frühling, H.E., Beato, C., Chinchilla, L., Llorente M.V. y De Misquita, P. (2004). Calles más seguras. Estudios de policía comunitaria en América Latina. En H. Frühling. (Ed), Reinventar la policía: la experiencia de Bello Horizonte (pp. 1-139). Washington D.C.: Banco Interamericano de desarrollo.

Frühling, H. (2009). Luces y Sombras en la reforma policial Latinoamericana. Revista Análisis Político, 2 (3). Recuperado de https://www.kas.de/c/document_library/get_ file?uuid $=04 f 4$ ed $81-0$ a94-bf76-04f8-aab0e3 cbdbe9\& groupId $=275611$

Fuquen, M.E. (2003). Los conflictos y las formas alternativas de resolución. Revista Tabula Rasa, $1,265-278$.

Galindo, J. (1998). Técnicas de investigación en sociedad, cultura y comunicación. Naucalpan de Juárez, México: Editorial Pearson.

González, L. y Delgado, C. (2018). Homicidio de defensores y defensoras de paz, una tragedia que no se detiene. Recuperado de https:/ / co.boell.org/sites/default/files/20180228_ideasverdes_ no6_web.pdf

Habermas, J. (2002). Teoría de la acción comunicativa, I, Racionalidad de la acción y racionalización social. Ciudad de México, México: Taurus.

Hernández-Sampieri, R., Fernández, C. y Baptista L.P. (2010). Metodología de la Investigación. México D. F., México: Mc Graw-Hill Companies.

Ley 1421 del 2010. (21 de diciembre), Por medio del cual se prorroga la 418 de 1997, prorrogada y modificada por las leyes 548, de 1999, 782 de 2002, 1106 de 2006. Diario oficial N. 47.930

Londoño, F. (2011). Esbozo de una teoría general de la ciencia de policía. Bogotá, Colombia: Policía Nacional de Colombia.

Maldonado, A. S. (2012). Drogas, violencia y militarización en el México rural. El caso de Michoacán. Revista Mexicana de Sociología, 74 (1), 5-39.

Mejía, R. (2008). La sistematización empodera y produce saber y conocimiento sobre la práctica desde la propuesta para sistematizar la experiencia de habilidades para la vida. Bogotá, Colombia: Ediciones Desde Abajo. 
Aciertos y desaciertos del servicio de policía en el ámbito rural del departamento de Santander, Colombia

Montañez, G. (2016). Territorios para la paz en Colombia: procesos entre la vida y el capital. Bitacora, 26, 11-28.

Oviedo, E. (2007). Modernización policial: el caso de Carabineros de Chile. Revista Latinoamericana de Seguridad Ciudadana, 71-84.

Ramírez, G. M. y Céspedes, E. (2016). Rol mediador de la policía nacional de Colombia en el posconflicto: un estudio del discurso de expertos con AEDT. Bogotá, Colombia: Escuela de Cadetes de Policía "General Francisco de Paula Santander”.

Ribeiro, L., Neiva e Oliveira, V. y Alves Diniz, A.M. (2016). Los significados de "policía comunitaria” para la Policía Militar Brasileña. Estudios Sociológicos, XXXIV, 603-637.

Rodríguez, J.A. (2015) • ¡Pan o palo! El perfil social de los jefes de policía rural en Tamaulipas, 1888-1910. Revista de El Colegio de San Luis, 222-242.

Ruíz, J.C. (2004). La encrucijada de la seguridad ciudadana en América Latina, entre la tentación autoritaria y la participación comunitaria. Recuperado de http://www.urosario.edu.co/ urosario_files/c9/c919a37d-16ba-401e-bbf5-7f6084996e41.pdf

Santos, J., Rivera, R., Naranjo, O., Adolfo, G., Páez, O. y León, J. (2010). Política para la consolidación del servicio de policía en el ámbito rural. Recuperado de http: / /www.policia. edu.co/documentos/tomos/SEGURIDAD\%20DE\%20LA\%20POLICIA\%20RURAL. pdf

Torres, J.M. (2015). ¿Es necesaria una nueva policía rural para el postconflicto? Recuperado de http: / / www.ideaspaz.org/publications/posts/1147

Villegas, L.C., Nieto J. H., Restrepo R. A., Mena, C.R., Rodríguez, J.E., González, R. y Blanco, R. (2016). Juntos por el Campo. Bogotá D. C., Colombia: Policía Nacional y Seguridad Ciudadana en el ámbito rural. Sistema Integrado de Seguridad Rural. SISER. Analecta de Doctrina OFPLA-CENPO, Edición impresa No 3, Policía Nacional, Oficina de Planeación Centro de Pensamiento y Doctrina Policial.

Weber, M. (1994). Economía y sociedad: fundamentos da sociología comprensiva. Brasilia, Brasil: Editora Universidad de Brasilia. 